\title{
Reform and Practice of Immersive Teaching in Environmental Acoustics
}

\author{
Haitao Wang*, Xiangyang Zeng, Ye Lei, Shuwei Ren, Xiaoyan Zhang \\ School of Marine Science and Technology, Northwestern Polytechnical University, Xi'an 710072, Shaanxi Province, China \\ *Corresponding author: Haitao Wang, wht@nwpu.edu.cn

\begin{abstract}
Environmental Acoustics is a professional course that educates students majoring in Acoustics. Affected by practical equipment and other factors, the teaching effect of knowledge points relevant to subjective auditory perception contained in the course is poor. Taking the course of Spatial Hearing and 3D Stereo as an example, this study develops a virtual simulation experiment system for the topic of subjective hearing perception in order to carry out the reform and practice of immersive teaching. Combined with the virtual simulation experiment project with high sense of presence, students are supported by the actual auditory perception effect in the whole learning process and have achieved good learning effect.
\end{abstract}

Keywords: Environmental Acoustics; Virtual simulation experiment; Immersive teaching

Publication date: December 2021; Online publication: December 23, 2021

\section{Introduction}

Acoustic processing and perception in different spaces is an important research branch in the field of acoustics and plays an important role in the field of national economy. For example, the noise source identification of cabins, the acoustic design of large halls, and the prediction of environmental noise have a direct impact on the noise control. The rapid development of related fields and the demand for scientific and technological innovation have raised the requirements of employees in terms of professional quality. It puts forward higher requirements in the training of students of related majors in universities.

At present, there are many studies on environmental engineering education and teaching in China, but there are only a few professional teachings characterized by environmental acoustics. Combined with the current discipline development trend of Environmental Acoustics, Northwestern Polytechnical University has introduced a series of courses under environmental acoustics for training, such as Acoustic Signal Processing, Engineering Acoustics, Sound Source Location and Identification, Spatial Hearing and 3D Stereo, as well as Noise Control Engineering. These courses play supporting roles for the training of students in this major. However, the feedbacks from students and graduates in recent years have revealed that some problems in the teaching need to be improved. These problems mainly focus on the contents of subjective auditory perception, which include auditory masking effect and human auditory positioning principle. Students do not experience the actual subjective auditory perception effect in the traditional theoretical teaching and practical design. In order to solve this problem, based on the practice of virtual simulation, the teaching group has designed and developed a personalized subjective auditory perception virtual simulation experiment, and based on this experiment, the reform of the teaching method with experiential teaching as the main way has been carried out.

This study introduces the design and development of a virtual simulation practice of subjective auditory perception and the experiential teaching method based on it; subsequently, it introduces the application and effect analysis of the new teaching method and practice in Spatial Hearing and 3D Stereo. 


\section{Immersive teaching based on the virtual simulation experiment of subjective auditory perception}

Taking Spatial Hearing and 3D Stereo as the platform, the subjective auditory perception virtual simulation experiment project is designed and developed from the personalized practice content of relevant knowledge points and then the reform of immersive teaching is carried out and applied to the course teaching.

\subsection{Development of subjective auditory perception virtual simulation experiment project}

Among all kinds of practical teaching methods, virtual simulation experiment is a new form of practical education that is rising in recent years. Virtual simulation experiment mainly relies on computer simulation to realize the simulation of objective physical phenomena and various principles. This changes the traditional experimental teaching method and breaks through the space-time limitation of experimental operations ${ }^{[1]}$. With its strong display characteristic, it has occupied an important position in practical teaching ${ }^{[2,3]}$. By combing the contents of the Spatial Hearing and 3D Stereo course and analyzing the curriculum difficulties reflected by previous students, the construction requirements of four virtual simulation experiments based on subjective auditory perception have been determined. According to this demand analysis, an auditory perception experimental system has been designed and developed, including four experimental contents: masking effect, Haas effect, Binaural auditory positioning, and spatial sound simulation.

The development of the virtual simulation experiment of subjective auditory perception takes the simple operation of students as the basic requirement. At the same time, in order to enable students to have their own subjective auditory experience, another basic requirement of the project is that students can freely input reasonable parameters and conduct personalized experiments. According to the above basic requirements, a schematic diagram of the design and the development process of the experimental project is shown in Figure 1.

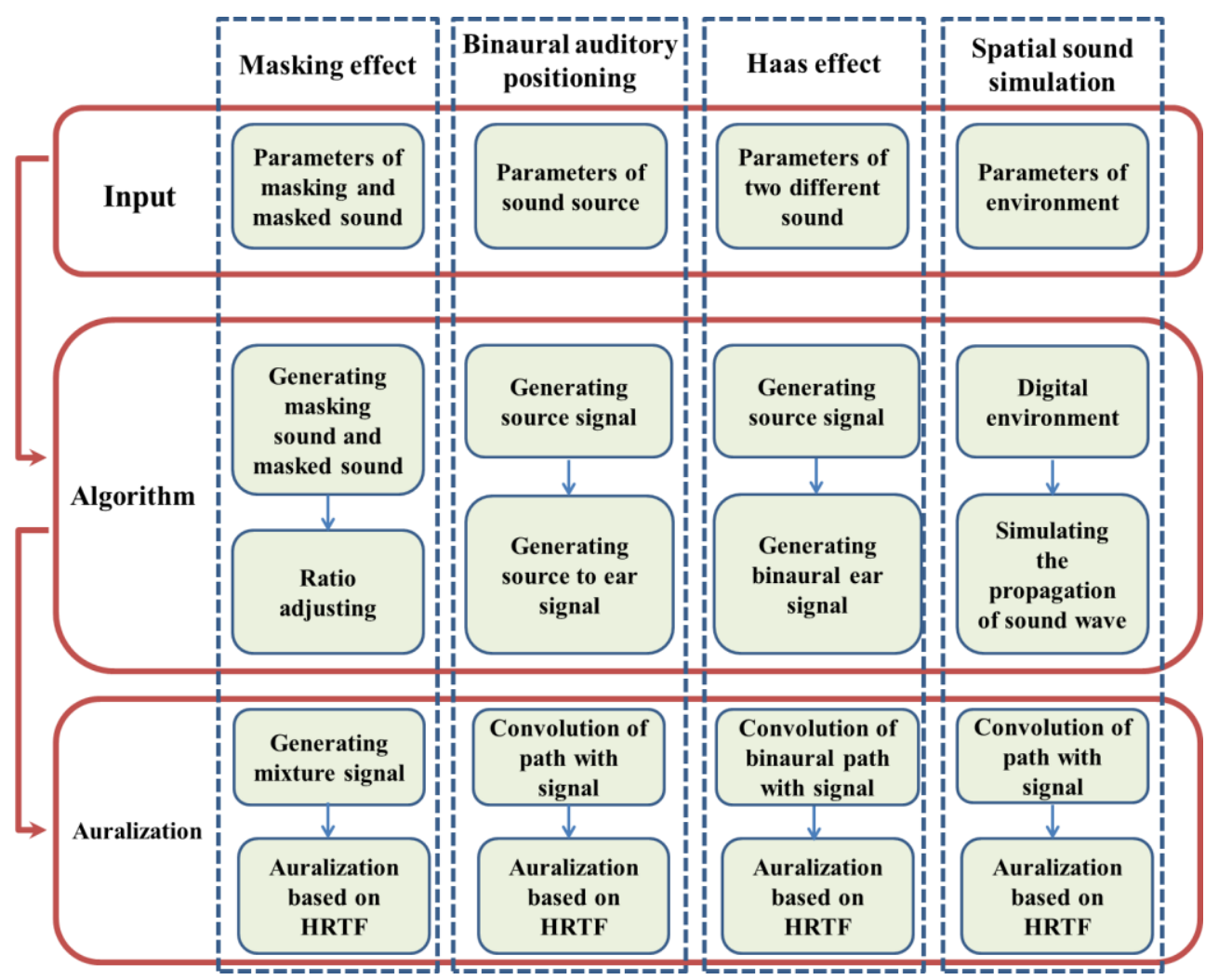

Figure 1. Design of virtual simulation experiment 
Figure 1 shows the design and development process of each experimental project. The experimental project is divided into three modules on the kernel: user parameter input, algorithm operation, and audible processing. The user parameter input module allows students to input arbitrary parameters within a reasonable range to realize their personalized experimental requirements. The algorithm operation module is the core module of the experimental system. According to the existing mature algorithms, virtual simulation algorithms such as virtual sound source, sound wave virtual propagation, and virtual receiver are designed and developed, thus completing the simulation of acoustic phenomena under various input parameters. Finally, in the audible processing module, based on the convolution technology of head correlation transfer function, the obtained virtual acoustic propagation process is used to complete the audible processing of the signal, and the results are visualized by means of field cloud drawing, as illustrated in Figure 2. Upon inputting parameters according to the above process, the audible signal can be directly obtained, and the subjective listening experience consistent with the real scene can be realized by using headphones or other equipment.
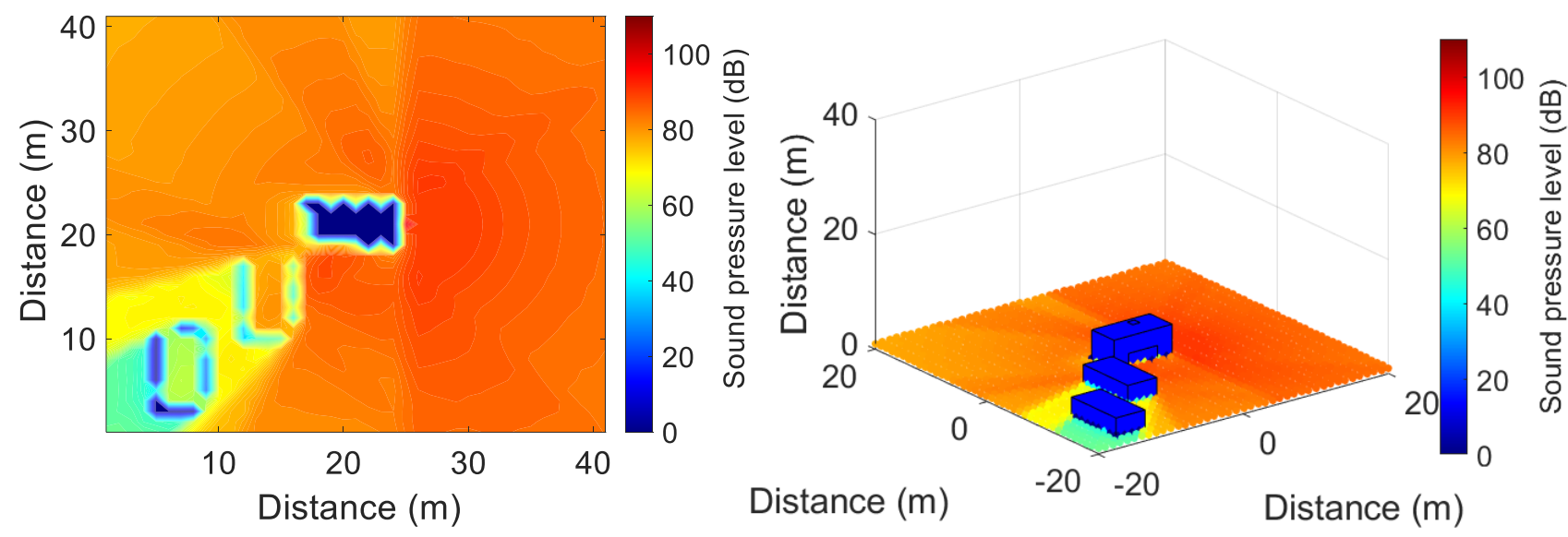

Figure 2. Simulating results of the experiment

\subsection{Reform of the teaching method based on virtual simulation experiment}

The traditional teaching method of Environmental Acoustics generally emphasizes on imparting knowledge and students' acceptance. In teaching, students' thinking and cognitive activities show one-way fluidity. Although this method results in good teaching effect of some knowledge points, it has a poor effect on contents that are closely related to people's subjective hearing. Therefore, relying on the virtual simulation experiment platform of subjective auditory perception, the reform of immersive teaching is carried out.

The prototype of immersive teaching began from the field of language teaching in the 1960s; in order to obtain good teaching results, students were completely immersed in the foreign language environment through the coordination of environment and teachers. With the continuous expansion of the application scale, a professor of psychology at the University of Chicago summarized and improved the immersive teaching method with the help of the immersion theory in the field of psychology, established the "immersive teaching" theory ${ }^{[4]}$, and used it in the field of engineering education ${ }^{[5-7]}$. With the development of virtual simulation technology, it allows the integration of scenes, images, and interactive information presented by virtual technology into classroom teaching, which doe not only bring great immersion experience to students, but also improve the teaching effect. Therefore, immersive teaching has greatly developed in recent years. 


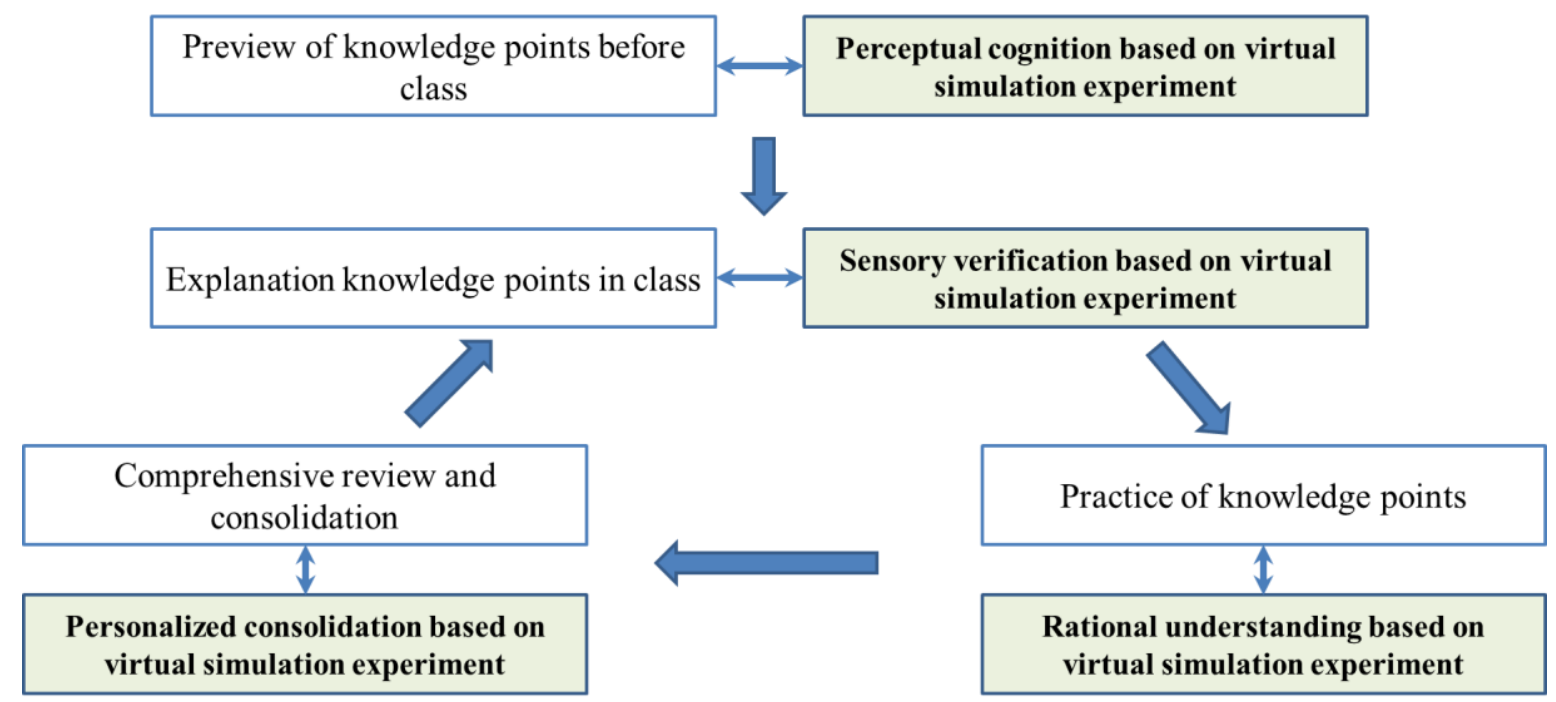

Figure 3. Flow chart of immersion teaching

In the immersive teaching design shown in Figure 3, students would gain preliminary understanding of relevant theories through the preview of knowledge points and the perceptual understanding of relevant acoustic phenomena with the help of virtual simulation experimental system prior to classes. The students would then enter the classroom teaching stage with problems. While the teacher completes the theory teaching in the classroom, students should be allowed to verify their knowledge with the help of auditory experiments. Through the practical guidance of teachers, the students' perceptual knowledge of acoustic phenomena can then be developed into a rational understanding. In the final comprehensive review and consolidation link, students would use the virtual simulation system to carry out personalized experiments to realize the complete understanding and mastery of knowledge points. In the above teaching process, the auditory practice of virtual simulation experiment based on subjective auditory perception runs through the whole teaching and constructs a personalized and situational classroom environment. Students would immerse themselves in it, assume the virtual simulation experiment as a support for theoretical learning, reshape the classroom into a dynamic classroom, and gain a wholehearted learning experience through interaction and reflection.

\section{Practice of immersive teaching}

In the spring semester of 2020 to 2021 academic year, immersive teaching has been implemented based on the subjective auditory perception virtual simulation experiment on the platform of the Spatial Hearing and $3 D$ Stereo course. The course consists of four chapters: basic acoustic theory, spatial auditory effect, human auditory positioning principle, and spatial sound quality simulation. In addition to the basic theory in Chapter 1, each chapter contains knowledge points related to subjective auditory perception.

Before each theoretical teaching, the teachers would arrange the preview tasks and allow the students to make preliminary perceptual understanding by using the subjective auditory perception virtual simulation experiment project through the operation instructions. During classes, the teachers would first gauge the understanding of the students from the preview and help the students master the difficulties encountered in the knowledge points. In the process of teaching, the focus is on explaining relevant knowledge points. At the same time, with the help of simulation experiments, students can preliminarily verify the accepted theoretical content. In practice, teachers use the virtual simulation system to demonstrate under different parameters, in order to help students gain a deeper understanding of the knowledge points. Students would then conduct personalized experimental operations to deepen their theoretical understanding from all 
aspects and consolidate those knowledge points. In the whole process of teaching and learning, the virtual simulation experiment of auditory perception practice is always placed in an auxiliary position in teaching. Through high-frequency perception practice, students can master the learned content through immersive experience. At the end of the course, a questionnaire survey was conducted among 23 students. The comparison of the degree of understanding of the knowledge points among the students before and after the educational reform is shown in Figure 4, where "1" on the transverse axis denotes "completely understand"; "2" denotes "partially understand"; "3" denotes "do not understand".
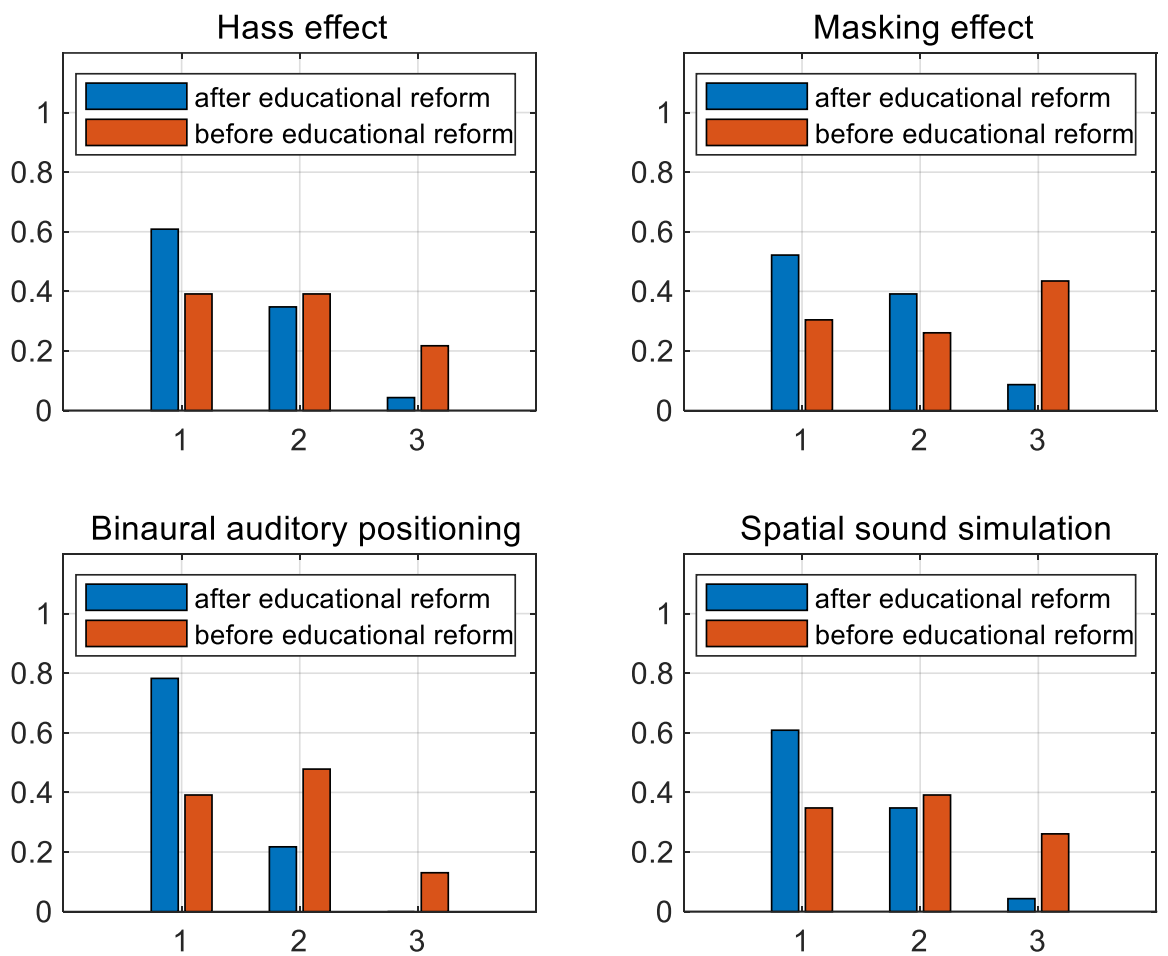

Figure 4. Comparison of the degree of understanding of the knowledge points before and after the practice of immersive teaching

As shown in Figure 4, it can be seen from the results that after implementing immersive teaching based on auditory perception virtual simulation experiment, the proportion of students who can fully understand the four knowledge points that have high correlation with subjective auditory perception has increased significantly, while the proportion of students who can only partially understand the content is low; this shows that the new immersive teaching method has achieved good teaching results in the comparison of two consecutive sessions.

\section{Conclusion}

Taking into consideration that there are many theoretical knowledge points of subjective auditory perception in Environmental Acoustics and the effect of traditional teaching methods is poor, a virtual simulation experimental system of auditory perception has been developed. This system can accurately simulate relevant acoustic phenomena and provide users with real auditory signals, so that the users can experience situational hearing. On this basis, the method of immersive teaching has been designed, so that students can be supported by their actual auditory experiences throughout the whole process of teaching, thus promoting their understanding of the knowledge points. The implementation of this method in the course of Spatial Hearing and 3D Stereo has proven its effectiveness. This teaching method would be 
widely used in the Environmental Acoustics course in the future and provide more reference to the development of virtual simulation practice in other disciplines.

\section{Funding}

This research was supported by the Key Teaching Reform Project of Northwestern Polytechnical University

- Practical Teaching Reform of Environmental Acoustics Course Group Supported by Virtual Auditory Perception Experiment Project (Project Number: 2021JGZ04).

\section{Disclosure statement}

The authors declare that there is no conflict of interest.

\section{Author contributions}

Haitao Wang and Xiangyang Zeng conceived the idea of the study. Shuwei Ren performed the experiments. Ye Lei and Xiaoyan Zhang analyzed the data and wrote the paper.

\section{References}

[1] Kang H, 2020, Construction of Typical Vehicle Use Environment based on Virtual Simulation Test. Journal of Physics: Conference Series, 1576(1): 012054. https://doi.org/10.1088/1742$6596 / 1576 / 1 / 012054$

[2] Liu S, 2012, Design and Parameterized Virtual Simulation Test of a Kind of Seat Bench for Ergonomic Test. Applied Mechanics and Materials, 215-216: 388-394. https://doi.org/10.4028/www.scientifi c.net/AMM.215-216.388

[3] Zhang W, Diao TS, 2021, On the attention management of college engineering students, Journal of Higher Education, 7(20): 85-88. https://kns.cnki.net/kcms/detail/detail.aspx?dbcode=CJFD\&dbname $=$ CJFDLAST2021\& filename $=$ GJXK202120020\&uniplatform $=$ NZKPT $\& v=m$ DyLIe4TNbLPXNYgS Vy6LNAdUjenY8aA\%25mmd2F\%25mmd2FGdycrSwxQOX3L0MHm8UgWA8HkBL\%25mmd2Fo $\mathrm{T}$

[4] Csikszentmihalyi M, 1975, Beyond Boredom and Anxiety: Jossey-Bass Publishers, San Francisco.

[5] Ceallaigh TJÓ, Máiréad H, Aisling L, 2021, Developing Potentiality: Pre-Service Elementary Teachers as Learners of Language Immersion Teaching. International Journal of Bilingual Education and Bilingualism, 24(4): 515-532. https://doi.org/10.1080/13670050.2018.1489779

[6] Li L, 2019, Enlightenment of Input Hypothesis and Output Hypothesis on English Teaching. Creative Education Studies, 7(2): 151-154. https://doi.org/10.12677/CES.2019.72027

[7] Stella K, Philip H, Chi Y, 2011, Immersion Education in China: Teachers' Perspectives. Frontiers of Education in China, 6(1): 68-91. https://doi.org/10.1007/s11516-011-0122-6 